\title{
External-Memory Multimaps
}

\author{
Elaine Angelino * \\ SEAS, Harvard University \\ elaine@eecs.harvard.edu \\ Michael T. Goodrich ${ }^{\dagger}$ \\ Department of Computer Science, University of California, Irvine, \\ goodrich@acm.org \\ Michael Mitzenmacher ${ }^{\ddagger}$ \\ SEAS, Harvard University \\ michaelm@eecs.harvard.edu \\ Justin Thaler ${ }^{\S}$ \\ SEAS, Harvard University \\ jthaler@seas.harvard.edu
}

\begin{abstract}
Many data structures support dictionaries, also known as maps or associative arrays, which store and manage a set of key-value pairs. A multimap is generalization that allows multiple values to be associated with the same key. For example, the inverted file data structure that is used prevalently in the infrastructure supporting search engines is a type of multimap, where words are used as keys and document pointers are used as values. We study the multimap abstract data type and how it can be implemented efficiently online in external memory frameworks, with constant expected I/O performance. The key technique used to achieve our results is a combination of cuckoo hashing using buckets that hold multiple items with a multiqueue implementation to cope with varying numbers of values per key. Our external-memory results are for the standard two-level memory model.
\end{abstract}

\section{Introduction}

A multimap is a simple abstract data type (ADT) that generalizes the map ADT to support key-value associations in a way that allows multiple values to be associated with the same key. Specifically, it is a dynamic container, $C$, of key-value pairs, which we call items, supporting (at least) the following operations:

- $\operatorname{insert}(k, v)$ : insert the key-value pair, $(k, v)$. This operation allows for there to be existing key-value pairs having the same key $k$, but we assume w.l.o.g. that the particular key-value pair $(k, v)$ is itself not already present in $C$.

- isMember $(k, v)$ : return true if the key-value pair, $(k, v)$, is present in $C$.

\footnotetext{
* To appear in ISAAC 2011. A preliminary version of this work appears as a Brief Announcement at SPAA 2011.

$\dagger$ Supported in part by the National Science Foundation under grants 0724806, 0713046, and 0847968, and by the Office of Naval Research under MURI grant N00014-08-1-1015.

$\doteqdot$ Supported in part by the National Science Foundation under grants 0915922 and 0964473.

${ }^{\S}$ Supported by the Department of Defense (DoD) through the National Defense Science \& Engineering Graduate Fellowship (NDSEG) Program, and partially by NSF grant CNS-0721491.
} 
- remove $(k, v)$ : remove the key-value pair, $(k, v)$, from $C$. This operation returns an error condition if $(k, v)$ is not currently in $C$.

- findAll $(k)$ : return the set of all key-value pairs in $C$ having key equal to $k$.

- removeAll $(k)$ : remove from $C$ all key-value pairs having key equal to $k$.

- $\operatorname{count}(k)$ : Return the number of values associated with key $k$.

Surprisingly, we are not familiar with any previous discussion of this abstract data type in the theoretical algorithms and data structures literature. Nevertheless, abstract data types equivalent to the above ADT, as well as multimap implementations, are included in the C++ Standard Template Library (STL) [17], Guava-the Google Java Collections Library 1$]$ and the Apache Commons Collection 3.2.1 AP ${ }^{2}$ Clearly, the existence of these implementations provides empirical evidence for the usefulness of this abstract data type.

\subsection{Motivation}

One of the primary motivations for studying the multimap ADT is that associative data in the real world can exhibit significant non-uniformities with respect to the relationships between keys and values. For example, many real-world data sets follow a power law with respect to data frequencies indexed by rank. The classic description of this law is that in a corpus of natural language documents, defined with respect to $n$ words, the frequency, $f(j, n)$, of the word of rank $j$ is predicted to be

$$
f(j, n)=\frac{1}{j^{s} H_{N, s}},
$$

where $s$ is a parameter characterizing the distribution and $H_{n, s}$ is the $n$th generalized harmonic number. Thus, if we wished to construct a data structure that can be used to retrieve all instances of any query word, $w$, in such a corpus, subject to insertions and deletions of documents, then we could use a multimap, but would require one that could handle large skews in the number of values per key. In this case, the multimap could be viewed as providing a dynamic functionality for a classic static data structure, known as an inverted file or inverted index (e.g., see Knuth [11]). Given a collection, $\Gamma$, of documents, an inverted file is an indexing strategy that allows one to list, for any word $w$, all the places in $\Gamma$ where $w$ appears.

Another powerful motivation for studying multimaps is graphical data [3]. A multimap can represent a graph: keys correspond to nodes, values correspond to neighbors, findAll operations list all neighbors of a node, and removeAll operations delete a node from the graph. The degree distribution of many real-life graphs follow a power law, motivating efficient handling of non-uniformity.

As a more recent example, static multimaps were used for a geometric hashing implementation on graphical processing units in [2]. In this setting, signatures are computed from an image, and a signature can appear multiple times in an image. The signature is a key, and the values correspond to locations where the signature can be found. Geometric hashing allows one to find query images within reference images. Dynamic multimaps could allow for changes in reference images to be handled dynamically without recalculating the entire structure.

There are countless other possible scenarios where we expect multimaps can prove useful. In many settings, one can indicate the intensity of an event or object by a score. Examples include the apparent brightness of stars (measured by stellar magnitudes), the intensity of earthquakes (measured on the Richter

1 http://code.google.com/p/google-collections/

2 http://commons.apache.org/collections/apidocs/index.html 
scale), and the loudness of sounds (measured on the decibel scale). Necessarily, when data from such scoring frameworks is labelled as key-value pairs where the numeric score is the key, some scores will have disproportionally many associated values than others. In fact, in assigning numeric scores to observed phenomena, there is a natural tendency for human observers to assign scores that depend logarithmically on the stimuli. This perceptual pattern is so common it is known as the Weber-Fechner Law [7, 10]. Multimaps may prove particularly effective for such data sets.

\subsection{Previous Related Work}

Inverted files have standard applications in text indexing (e.g., see Knuth [11]), and are important data structures for modern search engines (e.g., see Zobel and Moffat [23]). Typically, this is a static structure and the collection $\Gamma$ is usually thought of as all the documents on the Internet. Thus, an inverted file is a static multimap that supports the findAll( $(w)$ operation (typically with a cutoff for the most relevant documents containing $w$ ).

Cutting and Pedersen [6] describe an inverted file implementation that uses B-trees for the indexing structure and supports incremental and batched insertions, but it doesn't support deletions efficiently. More recently, Luk and Lam [14] describe an in-memory inverted file implementation based on hash tables with chaining, but their method also does not support fast deletions. Likewise, Lester et al. [12, 13] and Büttcher et al. [5] describe out-of-core inverted file implementations that support insertions only. Büttcher and Clarke [4], on the other hand, consider the trade-offs for allowing for both insertions and deletions in an inverted file, and Guo et al. [9] describe a solution for performing such operations by using a type of B-tree.

Our work utilizes a variation on cuckoo hash tables. We assume the reader has some familiarity with such hash tables, as originally presented by Pagh and Rodler [18] $]^{3}$ We describe the relevant background in Section 2 ,

Finally, recent work by Verbin and Zhang [21] shows that in the external memory model, for any dynamic dictionary data structure with query cost $O(1)$, the expected amortized cost of updates must be at least 1. As explained below, this implies our data structure is optimal up to constant factors.

\subsection{Our Results}

In this paper we describe efficient external-memory implementations of the multimap ADT. Our externalmemory algorithms are for the standard two-level I/O model, which captures the memory hierarchy of modern computer architectures (e.g., see [1, 22]). In this model, there is a cache of size $M$ connected to a disk of unbounded size, and the cache and disk are divided into blocks, where each block can store up to $B$ items. Any algorithm can only operate on cached data, and algorithms must therefore make memory transfer operations, which read a block from disk into cache or vice versa. The cost of an algorithm is the number of I/Os required, with all other operations considered free. All of our time bounds hold even when $M=O(B)$, and we therefore omit reference to $M$ throughout.

We support an online implementation of the multimap abstract data type, where each operation must completely finish executing (either in terms of its data structure updates or query reporting) prior to our beginning execution of any subsequent operations. The bounds we achieve for the multimap ADT methods are shown in Table 1. All bounds are unamortized.

Our constructions are based on the combination of two external-memory data structures-externalmemory cuckoo hash tables and multiqueues - which may be of independent interest. We show that external-

\footnotetext{
${ }^{3}$ A general description can be found on Wikipedia at http://en.wikipedia.org/wiki/Cuckoo_hashing.
} 


\begin{tabular}{|c|c|}
\hline Method & I/O Performance \\
\hline $\operatorname{insert}(k, v)$ & $\bar{O}(1)$ \\
\hline isMember $(k, v)$ & $O(1)$ \\
\hline $\operatorname{remove}(k, v)$ & $O(1)$ \\
\hline $\operatorname{findAll}(k)$ & $O\left(1+n_{k} / B\right)$ \\
\hline $\operatorname{removeAll}(k)$ & $O(1)$ \\
\hline $\operatorname{count}(k)$ & $O(1)$ \\
\hline
\end{tabular}

Table 1: Performance bounds for our multimap implementation. $\bar{O}(*)$ denotes an expected bound. Also, we use $B$ to denote the block size, $N$ to denote the number of key-value pairs, and $n_{k}$ to denote the number of key-value pairs with key equal to $k$.

memory cuckoo hashing supports a cuckoo-type method for insertions that provably requires only an expected constant number of I/Os. We then show that this performance can be combined with expected constant I/O complexity for multiqueues to design a multimap implementation that has constant (unamortized) worst-case or expected I/O performance for most methods. Our methods imply that one can maintain an inverted file in external memory so as to support a constant expected number of I/Os for insertions and worst-case constant I/Os for look ups and item removal.

\section{External-Memory Cuckoo Hashing}

In this section, we describe external-memory versions of cuckoo hash tables with multiple items per bucket. The implementation we describe in this section is for the map ADT, where all key-value pairs are distinct. We show later in this paper how this approach can be used in concert with multiqueues to support multiple key-value pairs with the same key for the multimap ADT.

Cuckoo hash tables that can store multiple items per bucket have been studied previously, having been introduced in [8]. Generally the analysis has been limited to buckets of a constant size, $d$, where here size is measured in terms of the number of items, which in this context is a key-value pair in our collection, $C$. For our external-memory cuckoo hash table, each bucket can store $B$ items, where $B$ is a parameter defining our block size and is not necessarily a constant.

Formally, let $\mathcal{T}=\left(T_{0}, T_{1}\right)$ be a cuckoo hash table such that each $T_{i}$ consists of $\gamma n / 2$ buckets, where each bucket stores a block of size $B$, with $n=N / B$. (In the original cuckoo hash table setting, $B=1$.) One setting of particular interest is when $\gamma=1+\varepsilon$ for some (small) $\varepsilon>0$, so that space overhead of the hash table is only an $\varepsilon$ factor over the minimum possible. The items in $\mathcal{T}$ are indexed by keys and stored in one of two locations, $T_{0}\left[h_{0}(k)\right]$ or $T_{1}\left[h_{1}(k)\right]$, where $h_{0}$ and $h_{1}$ are random hash functions. (The assumption that the hash functions are random can be done away with using suitable realistic hash functions; see for example [8] for a discussion, or [16] for an alternative model.)

It should be clarified that, in some settings, the use of a cuckoo hash function may be unnecessary or even unwarranted. Indeed, if $B>c \log n$ for a suitable constant $c$ and $\gamma=1+\varepsilon$, we can use simple hash tables, with just one choice for each item, instead. In this case, with Chernoff and union bounds one can 
show that with high probability all buckets will fit all the items that hash to it, since the expected number of items per bucket will then be $B /(1+\varepsilon)$, and $B$ is large enough for strong tail bounds to hold. Cuckoo hashing here allows us to avoid such "wide block assumptions", giving a more general approach. In practice, also, across the full range of possible values for $B$ we expect cuckoo hashing to be much more space efficient. Whether this space savings is important may depend on the setting.

The important feature of the cuckoo hashing implementation is the way it may reallocate items in $\mathcal{T}$ during an insertion. Standard cuckoo hashing, with one item per bucket, immediately evicts the previous (and only) item in a bucket when a new item is to be inserted in an occupied bucket. With multiple items per bucket, there is a choice available. We describe what is known in this setting, and how we modify it for our use here.

Let $G$ be the cuckoo graph, where each bucket in $\mathcal{T}$ is a vertex and, for each item $x$ currently in $C$, we connect $T_{0}\left[h_{0}(x)\right]$ and $T_{1}\left[h_{1}(x)\right]$ as a directed edge, with the edge pointing toward the bucket it is not currently stored in. Suppose we wish to insert an item $x$ into bucket $X$ in $\mathcal{T}$. If $X$ contains fewer than $B$ items, then we simply add $x$ to $X$. Otherwise, we need to make room for the new item.

One approach for doing an insertion is to use a breadth first search on the cuckoo graph. The results of Dietzfelbinger and Weidling show that for sufficiently large constant $B$, the expected insertion time is constant. Specifically, when $\gamma=1+\varepsilon$ and $B \geq 16 \ln (1 / \varepsilon)$, the expected time to insert a new key is $(1 / \varepsilon)^{O(\log \log (1 / \varepsilon))}$, which is a constant. (This may require re-hashing all items in very rare cases when an item cannot be placed; the expected time remains constant.) Notice that if $B$ grows in a fashion that is $\Omega(1)$, then a breadth first search approach does not naturally take constant expected time, as even the time to look if items currently in the bucket can be moved will take $\Omega(B)$ time. (It might still take constant expected time - it may be that only a constant number of buckets need to be inspected on average - but it does not appear to follow from [8].)

For non-constant $B$, we can apply the following mechanism: we can use our buckets to mimic having $B / c$ distinct subtables for some large constant $c$, where the $i$ th subtable uses the $c i / B$ th fraction of the bucket space, and each item is hashed into a specific subtable. For $B=O\left(n^{\delta}\right)$ for $\delta<1$, each subtable will contain close to its expected number of items with high probability. Further, by choosing $c$ suitably large one can ensure that each subtable is within a $1+\varepsilon$ factor of its total space while maintaining an expected $\log (1 / \varepsilon)^{O(\log \log (1 / \varepsilon))}$ insertion time. Specifically, we have the following theorem:

Theorem 1. Suppose for a cuckoo hash table $\mathcal{T}$ the block size satisfies $B=\Omega(1)$ and $B=O\left(n^{\delta}\right)$ for $\delta<1$. Let $0<\varepsilon \leq 0.1$ be arbitrary, let $C$ be a collection of $N$ items, and let $T$ be a table with at least $(1+\varepsilon) N / B$ blocks. Suppose further we have B/c subtables, with $c=16 \ln (1 / \varepsilon)$ with each item hashed to a subtable by a fully random hash function, and the hash functions for each subtable are fully random hash functions. Finally, suppose the items of $C$ have been stored in $\mathcal{T}$ by an algorithm using the partitioning process described above and the cuckoo hashing process. Then the expected time for the insertion of a new item $x$ using a BFS is $(1 / \varepsilon)^{O(\log \log (1 / \varepsilon))}$.

Proof: Each subtable has the capacity to hold $(1+\varepsilon) N c / B$ items, and will receive an expected $N c / B$ items to store. Let $X$ be the number of items in the first subtable. A standard Chernoff bound (e.g., [15][Theorem 4.4]) gives that $X$ is at most $(1+\varepsilon / 3) N c / B$ with probability bounded by

$$
\operatorname{Pr}\left(X \geq\left(1+\frac{\varepsilon}{3}\right) \frac{N c}{B}\right) \leq e^{-N c \varepsilon^{2} /(27 B)} .
$$

With $B=O\left(n^{\delta}\right)$ for $\delta<1$, we see that all subtables have at most $(1+\varepsilon / 3) N c / B$ with probability subexponential in $N^{1-\delta}$. By keeping counters for each subtable, we can re-hash the items of all subtables in the rare 
case where a subtable exceeds this number of items without affecting the expected insertion time by more than an $o(1)$ term.

The proof follows from Theorem 2 of [8], by noting that each subtable has space for at least $(1+$ $\varepsilon / 2)(1+\varepsilon / 3) N c / B<(1+\varepsilon) N c / B$ items. (In rare cases where an insertion fails, we can re-insert all items in a subtable without affecting the expected insertion time by more than an $o(1)$ term.)

It is likely this result could be improved (see the remarks in [8]), but it is sufficient for our purposes of showing that there is an insertion method for external-memory cuckoo tables that uses a constant expected number of I/Os.

As noted in [8], a more practical approach is to use random walk cuckoo hashing in place of breadth first search cuckoo hashing. (For example, random walk cuckoo hashing is used in all experiments in [8].) With random walk cuckoo hashing, when an item cannot be placed, it kicks out a single item in the bucket chosen uniformly at random. Random walk cuckoo hashing avoids the potentially large rare memory overhead required of breadth first search, allowing instead a nearly stateless solution.

More specifically, suppose a bucket $X$ is full when placing an item $x$. To reallocate items, we perform a random walk on the buckets, starting from $X$, to find an augmenting path that has the net effect of freeing up a location in $X$ (for $x$ ) while maintaining the two-choice allocation rule for all the existing items in $C$. Let $Y$ denote the current node we are visiting in our random walk (which is associated with a full bucket in the external-memory cuckoo table-initially, the bucket $X$ ). To identify the next node to visit, we choose one of the items, $y$, in $Y$, uniformly at random. We then remove $y$ from $Y$ and insert the item $x$ waiting to be inserted in $Y$. We then let $y$ take over the role of $x$, and attempt to place $x$ in the other bucket that is a possible location for this item. We repeat this process until we find a non-full bucket or reach a pre-defined stopping condition.

For loads arbitrarily close to one, it is not known if there is a random walk cuckoo hashing scheme using two bucket choices and multiple items per bucket that similarly achieves expected constant insertion time and logarithmic insertion time with high probability. (This is given as an open question in [8].) Sadly, we do not resolve this question here.

However, for loads up to about $2 / 3$ we can utilize results by Panigrahy [19, 20] to obtain such a random walk cuckoo hashing scheme. In Theorem 2.3.2 of [20], he shows that for hash tables for $t$ items and load factors of $s$ satisfying $(2 s)\left(1-e^{-2 s}\right)<1$, when the bucket size is 2 , random walk cuckoo hashing will succeed in inserting an item with a path of length $O(\log t)$ with probability $1-O\left(1 / t^{2}\right)$; his argument also shows that this process has expected constant insertion time. This allows loads up to (approximately) $2 / 3$ using our partitioning technique above. In practice, we might expect this load to be improved significantly in various ways. First, we might ignore the partitioning, and instead perform the random walk directly on the buckets with load $B$. Analyzing this process is difficult, in part because of the greatly increased possibility of cycles in the cuckoo graph. Alternatively, we could perform the partitioning but allow the random walk to stop early if there is room in the block $B$, rather than the bucket for the corresponding subtable, effectively multiplexing the bucket over subtable instantiations. We consider these multiple variations in the simulations of Section 5 .

Finally, we point out that, as in a standard cuckoo hash table, item look ups and removals use a worstcase constant number of I/Os. 


\section{External-Memory Multimaps}

In this section, we describe an extension of the external-memory cuckoo hash table (as described in Section 2) that can be used to maintain a multimap in external memory, so as to support fast dynamic access of a massive data set of key-value pairs where some keys may have many associated values.

\subsection{The Primary Structure}

To implement the multimap ADT, we begin with a primary structure that is an external-memory cuckoo hash table storing just the set of keys. In particular, each record, $R(k)$, in $\mathcal{T}$, is associated with a specific key, $k$, and holds the following fields:

- the key, $k$, itself

- the number, $n_{k}$, of key-value pairs in $C$ with key equal to $k$

- a pointer, $p_{k}$, to a block $X$ in a secondary table, $\mathcal{S}$, that stores items in $C$ with key equal to $k$. Let $n_{k}$ denote the number of key-value pairs in $C$ with key equal to $k$. If $n_{k}<B$, then $X$ stores all the items with key equal to $k$ (plus possibly some items with keys not equal to $k$ ). Otherwise, if $n_{k} \geq B$, then $p_{k}$ points to a first block of items with key equal to $k$, with the other blocks of such items being stored elsewhere in $\mathcal{S}$.

This secondary storage is an external-memory data structure we are calling a multiqueue.

\subsection{An External-Memory Location-Aware Multiqueue}

\subsubsection{Overview}

The secondary storage that we need in our construction is a way to maintain a set $\mathcal{Q}$ of queues in external memory. We assume the header pointers for these queues are stored in an array, $\mathcal{T}$, which in our externalmemory multimap construction is the external-memory cuckoo hash table described above.

For any queue, $Q$, we wish to support the following operations:

- enqueue $(x, H)$ : add the element $x$ to $Q$, given a pointer to its header, $H$.

- remove $(x)$ : remove $x$ from $Q$. We assume in this case that each $x$ is unique.

- isMember $(x)$ : determine whether $x$ is in some queue, $Q$.

In addition, we wish to maintain all these queues in a space-efficient manner, so that the total storage is proportional to their total size. To enable this, we store all the blocks used for queue elements in a secondary table, $\mathcal{S}$, of blocks of size $B$ each. Thus, each header record, $H$ in $\mathcal{T}$, points to a block in $\mathcal{S}$.

Our intent is to store each queue $Q$ as a doubly-linked list of blocks from $\mathcal{S}$. Unfortunately, some queues in $\mathcal{Q}$ are too small to deserve an entire block in $S$ dedicated to storing their elements. So small queues must share their first block of storage with other small queues until they are large enough to deserve an entire block of storage dedicated to their elements. Initially, all queues are assumed to be empty; hence, we initially mark each queue as being light. In addition, the blocks in $\mathcal{S}$ are initially empty; hence, we link the blocks of $\mathcal{S}$ in a consecutive fashion as a doubly-linked list and identify this list as being the free list, $F$, for $\mathcal{S}$. 
We set a heavy-size threshold at $B / 3$ elements. When a queue $Q$ stored in a block $X$ reaches this size, we allocate a block from $\mathcal{S}$ (taking a block off the free list $F$ ) exclusively to store elements of $Q$ and we mark $Q$ as heavy. Likewise, to avoid wasting space as elements are removed from a queue, we require any heavy queue $Q$ to have at least $B / 4$ elements. If a heavy queue's size falls below this lower threshold, then we mark $Q$ as being light again and we force $Q$ to go back to sharing it space with other small queues. This may in turn involve returning a block to the free list $F$. In this way, each block $X$ in $\mathcal{S}$ will either be empty or will have all its elements belonging to a single heavy queue or as many as $O(B)$ light queues. In addition, these rules also imply that $O(B)$ element insertions are required to take a queue from the light state to the heavy state and $O(B)$ element removals are required to take a queue from the heavy state to the light state.

If a block $X$ in $\mathcal{S}$ is being used for light queues, then we order the elements in $X$ according to their respective queues. Each block for a heavy queue $Q$ stores previous and next pointers to the neighboring blocks in the linked list of blocks for $Q$, with the first such block pointing back to the header record for $Q$. As we show, this organization allows us to maintain our size and label invariants during execution of enqueue and remove operations.

One additional challenge is that we want to support the remove $(x)$ operation to have a constant I/O complexity. Thus, we cannot afford to search through a list of blocks of a queue looking for an element $x$ we wish to remove. So, in addition to the table $\mathcal{S}$ and its free list, $F$, and the headers for each queue in $\mathcal{Q}$, we also maintain an external-memory cuckoo hash table, $\mathcal{D}$, to be a dictionary that maps each queue element $x$ to the block in $\mathcal{S}$ that stores $x$. This allows our multiqueue to be location-aware, that is, to support fast searches to locate the block in $\mathcal{S}$ that is holding any element $x$ that belongs to some queue, $Q$.

We will call any block in $\mathcal{S}$ containing fewer than $B / 4$ items deficient. In order to ensure that our multiqueue uses total storage proportional to its total size, we will enforce the following two rules. Together, these rules guarantee that there are $O(N / B)$ deficient blocks in $\mathcal{S}$, and hence our multiqueue uses $O(N / B)$ blocks of memory.

1. Each block $Y$ in $\mathcal{T}$ stores a pointer $d$, called the deficient pointer, to a block $d(Y)$; the identity of this block is allowed to vary over time. We ensure that at all times, $d(Y)$ is the only (possibly) deficient block associated with $Y$ that stores light queues.

2. Each heavy queue $Q$ also stores in its header block a deficient pointer $d$ to a block $d(Q)$. At all times, $d(Q)$ is the only (possibly) deficient block devoted to storing values for $Q$.

\subsubsection{Full Description}

For the remainder of this subsection, we describe how to implement all multiqueue operations to obtain constant amortized expected or worst-case runtime. We show how to deamortize these operations in Section 3.3 .

The Split Action. As we perform enqueue operations, a block $X$ may overflow its size bound, $B$. In this case, we need to split $X$ in two, which we do by allocating a new block $X^{\prime}$ from $\mathcal{S}$ (using its free list). We call $X$ the source of the split, and $X^{\prime}$ the sink of the split. We then proceed depending on whether $X$ contains elements from light queues or a single heavy queue.

1. $X$ contains elements from light queues. We greedily copy elements from $X$ into $X^{\prime}$ until $X^{\prime}$ has size has size at least $B / 3$, keeping the elements from the same light queue together. Note that each light queue has less than $B / 3$ elements, so this split will result in at least a $1 / 3-2 / 3$ balance. 
Of course, to maintain our invariants, we must change the header records from $X$ to $X^{\prime}$ for any queues that we just moved to $X^{\prime}$. We can achieve this by performing a look-up in $\mathcal{T}$ for each key corresponding to a queue that was moved from $X$ to $X^{\prime}$, and modifying its header record, which requires $O(B)$ I/Os. Similarly, in order to support location awareness, we must also update the dictionary $\mathcal{D}$. So, for each element $x$ that is moved to $X^{\prime}$, we look up $x$ in $\mathcal{D}$ and update its pointer to now point to $X^{\prime}$. In total this costs $O(B) \mathrm{I} / \mathrm{Os}$.

2. $X$ contains elements from a single heavy queue $Q$. In this case, we move no elements, and simply take a block $X^{\prime}$ from the free list and insert it as the head of the list of blocks devoted to $Q$, changing the header record $H$ in $\mathcal{T}$ to point to $X^{\prime}$. We also change the deficient pointer $d$ for $Q$ to point to $X^{\prime}$, and insert into $X^{\prime}$ the element that caused the split. This takes $O(1) \mathrm{I} / \mathrm{O}$ operations in total.

So, to sum up, when a block holding light queues results from a split (source or sink), it has size at least $B / 3$ and at most $2 B / 3$. When a block holding elements from a heavy queue $Q$ is split, no items are moved and a block is taken from the free list and inserted as the new header block of the heavy queue; the new header then contains only one item, and is identified by the deficient pointer of $Q$.

The Enqueue Operation. Given the above components, let us describe how we perform the enqueue and remove operations. We begin with the enqueue $(x, H)$ operation. We consider how this operation acts, depending on a few cases.

1. The queue for $H$ is empty (hence, $H$ is a null pointer and its queue is light). In this case, we examine the block $Y$ from $\mathcal{T}$ to which $H$ belongs. If $d(Y)$ is null, we first take a block $X$ of the free list and set $d(Y)$ to $X$ before continuing. We follow the deficient pointer for $Y$ to a block $X^{\prime}$, and add $x$ to $X^{\prime}$. If this causes the size of $X^{\prime}$ to reach $B$, then we split $X^{\prime}$ as described above.

2. The queue $Q$ for $H$ is not empty. We proceed according to two cases.

(a) If $Q$ is a light queue, we follow $H$ to its block $X$ in $\mathcal{S}$ and add $x$ to $X$. If this brings the size of $Q$ above $B / 3$, we perform a light-to-heavy transition, taking a block $X^{\prime}$ off the free list, moving all elements in $Q$ to $X^{\prime}$, and marking $Q$ as heavy. If this brings the size of $X$ below $B / 4$, we process $X$ as in the remove operation below.

(b) If $Q$ is a heavy queue, we add $x$ to $X=d(Q)$, the (possibly) deficient block for $Q$. If this brings the size of $X$ to $B$, then we split $X$, as described above.

Once the element $x$ is added to a block $X$ in $\mathcal{S}$, we then add $x$ to the dictionary $\mathcal{D}$, and have its record point to $X$.

The Remove and isMember Operations. In both of these operations, we look up $x$ in $\mathcal{D}$ to find the block $X$ in $\mathcal{S}$ that contains $x$. In the isMember $(x)$ case, we complete the operation by simply looking for $x$ in $X$. In the remove $(x)$ operation, we do this look up and then remove $x$ from $X$ if we find $x$. If this causes $Q$ to become empty, then we update its header, $H$, to be null. In addition, if this operation causes the size of $X$ to go below $B / 4$, then we need to do some additional work, based on the following cases:

1. $Q$ is a heavy queue.

(a) If $X$ is the only block for $Q$, then $Q$ should now be considered a light queue; hence, we continue processing it according to the case listed below where $X$ contains only light queues. We refer to the entirety of this action as a heavy-to-light queue transition. 
(b) Otherwise, if $X=d(Q)$, then we are done because $d(Q)$ is allowed to be deficient. If $X \neq d(Q)$, we proceed based on the following two cases:

i. $d$-alteration action: If the size of $d(Q)$ is at least $2 B / 3$, we simply update $Q$ 's deficient pointer, $d$, to point to $X$ instead of $d(Q)$.

ii. Merge action: If the size of $d(Q)$ is less than $2 B / 3$, then we move all of the elements of $X$ into $d(Q)$ and we update the pointer in $\mathcal{D}$ for each moved element. $X$ is returned to the free list. We call $X$ the source of the merge, and $d(Q)$ the sink. (Note that in this case, the size of $d(Q)$ becomes at most $11 B / 12$.)

2. $X$ contains light queues (hence, no heavy queue elements). In this case, we visit the header $H$ for $Q$. Let $Y$ denote the block containing $H$.

(a) If $X=d(Y)$ we are done, since $d(Y)$ is allowed to be deficient.

(b) If $X \neq d(Y)$, let $Z$ be the size of $d(Y)$.

i. $d$-alteration action: If $Z \geq 2 B / 3$ then we simply update $d$ to point to $X$ instead of $d(Y)$.

ii. Merge action: If $Z<2 B / 3$, then we merge the elements in $X$ into $d(Y)$, which now has size at most $11 B / 12$, and update pointers in $\mathcal{D}$ and $\mathcal{T}$ for the elements that are moved. We return $X$ to the free list. We call $X$ the source of the merge and $d(Y)$ the sink.

If a block $X^{\prime}$ is pointed to by any deficient pointer $d$, it is helpful to think of this as "protection" for $X^{\prime}$ from being the source of a merge. Once $X^{\prime}$ is afforded this protection, it will not lose it until its size is at least $2 B / 3$ (see the $d$-alteration action). At a high level, this will allow us to argue that if $X$ and $X^{\prime}$ are respectively the source and sink of a merge action, neither $X$ nor $X^{\prime}$ will be the source of a subsequent merge or split operation until it is the target of $\Omega(B)$ enqueue or remove operations, even though $X^{\prime}$ may have size very close to the deficiency threshold $B / 4$.

\subsubsection{Amortized I/O complexity}

We now argue formally that enqueue $(x, H)$ and remove $(x)$ take $O(1)$ amortized time. Notice that the only actions that result in the movement of items between blocks are light-to-heavy and heavy-to-light queue transitions, merge actions, and split actions for blocks containing light queues. Notice for splits involving heavy queues, we perform $O(1) \mathrm{I} / \mathrm{O}$ operations in the worst case, and do not need to perform an amortized analysis.

We first argue that light-to-heavy queue transitions as well as heavy-to-light transitions contribute $O(1)$ amortized I/Os to enqueue operations. Indeed, a light-to-heavy queue transition requires $O(B) \mathrm{I} / \mathrm{Os}$ in total: we require $O(B) \mathrm{I} / \mathrm{Os}$ to move $O(B)$ items from $X$ to $X^{\prime}$ and update pointers in $\mathcal{D}$ and $\mathcal{T}$, and $O(B)$ additional I/Os to process $X$ as in a remove operation if this causes the size of $X$ to fall below $B / 4$. Each such heavy-tolight transition must be preceded by at least $B / 12$ enqueue operations to bring the queue from size at most $B / 4$ to size at least $B / 3$, so we can charge these $O(B) \mathrm{I} / \mathrm{Os}$ to these enqueue operations. These enqueue operations will never be charged again. Similarly, a heavy-to-light queue transition requires $O(B) \mathrm{I} / \mathrm{Os}$, which we can charge to the (at least) $B / 12$ removals that caused $Q$ 's size to fall from $B / 3$ to $B / 4$; these removals will never be charged again.

Since we have accounted for the I/Os caused by light-to-heavy and heavy-to-light queue transitions, we may ignore all I/Os caused by these transitions through the remainder of the argument. We now argue that merge and split actions contribute $O(1)$ amortized I/Os as well, beginning with merge actions. 
Suppose $X$ and $X^{\prime}$ are respectively the source and sink of a merge action. We claim that neither $X$ nor $X^{\prime}$ will be the source of a subsequent merge or split operation until it is the target of $\Omega(B)$ enqueue or remove operations. Indeed, notice that after doing a merge action as a part of our processing of a remove operation, the sink will contain at most $11 B / 12$ elements and will be equal to $d(Y)$ or $d(Q)$, and the source is on the free list. As $d(Y)$ and $d(Q)$ are protected from merges, it would take at least $B / 12$ enqueues or removals in these blocks before they would be sources of another split or merge operation.

Likewise, after performing a split of a block containing light queues as a part of an enqueue operation, both source and sink will be of size at least $B / 3$ and at most $2 B / 3$. Thus, it would take at least $B / 12$ enqueues or removals in these blocks before they would be sources of another split or merge operation.

Therefore, in an amortized analysis, we can charge the $O(B) \mathrm{I} / \mathrm{Os}$ performed in a split or merge action to the previous $O(B)$ operations that caused one of these blocks to shrink to size $B / 4$ or grow to size $B$. These enqueues and removals will never be charged again.

The arguments of the last two paragraphs are depicted graphically in Figure 1. Assuming no light-toheavy or heavy-to-light transitions take place (we may assume this because we have separately accounted for the I/O cost of these transitions), we depict a subgraph of the state diagram for any block $X$. Specifically, we depict all state transitions caused by any action that results in the movement of items from one block to another; for brevity, we omit the effects of any actions that do not result in the movement of items. We refer to any state corresponding to a source of a merge or split action as a "source state." It is clear that in the subgraph depicted in Figure 11, there is no directed path from any non-source state to any source state. Given this fact, it is a straightforward exercise to confirm that the only paths from non-source states to source states in the full state diagram (assuming no light-to-heavy or heavy-to-light transitions) include at least $B / 12$ enqueue or remove operations to $X$.

\subsection{Deamortizing Multiqueue Operations}

We now explain how to deamortize the multiqueue operations of the previous section. First, notice that the only actions that result in the movement of items between blocks are merge actions, split actions for blocks devoted to heavy queues, light-to-heavy queue transitions, and heavy-to-light queue transitions. We will require the follow property: for any action resulting in the movement of items from source block $X$ to sink block $X^{\prime}$, neither $X$ nor $X^{\prime}$ will be the source of any subsequent action requiring the movement of items until it is the target of at least $B / 12$ enqueue or remove operations.

First, we describe some modifications to the light-to-heavy and heavy-to-light queue transitions that are necessary to ensure this property is satisfied. We begin with light-to-heavy transitions. Previously, as soon as a light queue $Q$ grew to size $B / 3$, it was moved from its block $X$ to a block $X^{\prime}$ devoted exclusively to $Q$; this could cause the size of $X$ to fall close to or below $B / 4$, and $X$ could therefore be the source of a merge shortly after (or immediately upon) the light-to-heavy transition. Because this clearly does not satisfy the required property, we will do away with an explicit light-to-heavy transition action, and instead fold this functionality into the split action as follows.

We leave unmodified the split action for blocks $X$ devoted to heavy queues, as well as for blocks $X$ containing only light queues in which none of the queues have size greater than $B / 3$. It is easy to see in both of these cases that the required property is satisfied, as in the first case (split for blocks devoted to heavy queues) no items are moved, and in the second case both the source and sink of the split have size between $B / 3$ and $2 B / 3$.

However, if the source $X$ of the split move contains a queue $Q$ of size at least $B / 3$, we proceed according to the following cases. 


\begin{tabular}{c|c}
$S \leq 2 B / 3$, \\
$X=d(Q)$
\end{tabular}$\quad \begin{gathered}\text { merge } \\
\text { sink }\end{gathered} \quad \begin{gathered}S \leq \frac{11 B}{12} \text { and } \\
X=d(Q)\end{gathered}$

$X$ contains heavy queues $X$ contains heavy queues

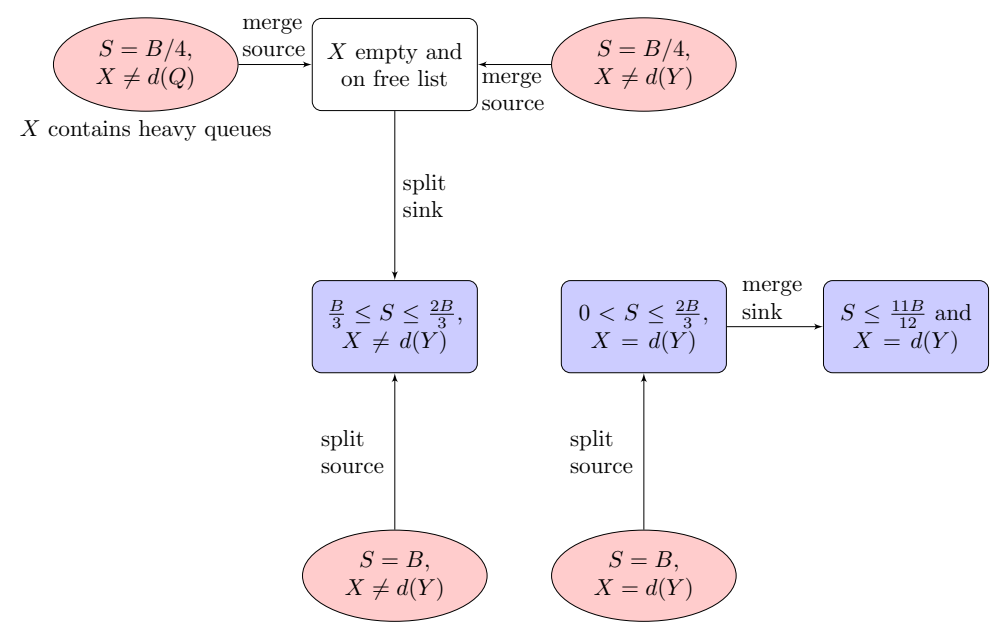

Figure 1: A subgraph of the state diagram for any block $X$, depicting all state transitions caused by merge or split actions. $S$ denotes the size of $X$. Ovals denote source states, while rectangles denote non-source states. Unless otherwise noted, any state depicted is for a block containing light queues. 
1. $X$ contains a queue $Q$ of size between $B / 3$ and $2 B / 3$. We take a new block $X^{\prime}$ off the free list and move all items in $Q$ to $X^{\prime}$, marking $Q$ as heavy and updating the affected pointers in $\mathcal{T}$ and $\mathcal{D}$. After this split action, both $X$ and $X^{\prime}$ have size between $B / 3$ and $2 B / 3$, and hence neither will be the source of a split action or merge action until it is the target of at least $B / 12$ enqueue or remove operations.

2. $X$ contains a queue $Q$ of size greater than $2 B / 3$. Let $\mathcal{I}$ denote the items in $X$ that are not in $Q$. We proceed according to the following cases.

(a) If $d(Y)$ has size less than $B / 3$, we leave $Q$ in $X$ and mark it as heavy. In addition, we transfer all items in $\mathcal{I}$ to $d(Y)$, and update all affected pointers in $\mathcal{T}$ and $\mathcal{D}$. After the split, $X$ is devoted to $Q$ and has size at least $2 B / 3 . X^{\prime}$ now has size at most $2 B / 3$, and moreover $X^{\prime}=d(Y)$ and thus $X^{\prime}$ is protected from being the source of a merge. It therefore requires at least $B / 12$ inserts or removals to $X$ or $X^{\prime}$ before either can be the source of any action requiring the movement of items between blocks.

(b) If $d(Y)$ has size greater than $B / 3$, we leave $Q$ in $X$ and mark it as heavy. We take a new block $X^{\prime}$ off the free list and transfer all items in $\mathcal{I}$ to $X^{\prime}$. We update all affected pointers in $\mathcal{T}$ and $\mathcal{D}$, and modify the deficient pointer $d$ of $Y$ to point to $X^{\prime}$. The source block $X$ is devoted to $Q$ and has size at least $2 B / 3 . X^{\prime}$ has size $|\mathcal{I}| \leq B / 3$, and moreover $X^{\prime}=d(Y)$ and thus $X^{\prime}$ is protected from being the source of a merge. It therefore requires at least $B / 12$ inserts or removals to $X$ or $X^{\prime}$ before either can be the source of any subsequent action requiring the movement of items between blocks.

Let us now explain a small modification we must make to the heavy-to-light transitions in order to satisfy the required property. Observe that it is possible for a queue $Q$ to undergo a heavy-to-light transition shortly after the final two blocks $X$ and $X^{\prime}$ devoted to $Q$ are merged into one. For example, it is possible that $X^{\prime}=d(Q)$ contains one item before the merge and $B / 4+1$ items after the merge; if one item is subsequently removed from $X^{\prime}, Q$ will undergo a heavy-to-light transition, and our required property will not be satisfied. This is the only setting in which a deficient pointer fails to "protect" a block from being merged. To circumvent this difficulty, we modify the heavy-to-light queue transition to only occur when the size of the heavy queue falls below $B / 6$ rather than $B / 4$. With this in hand, the arguments of Section 3.2 .3 suffice to show that any merge action or heavy-to-light transition satisfies our required property. This completes the description of all modifications necessary to ensure the required property is satisfied by all actions.

We now explain how to deamortize the operations of Section 3.2, which all required $O(1)$ amortized time. The only actions requiring $\omega(1) \mathrm{I} / \mathrm{O}$ operations in Section 3.2 were split actions, merge actions, heavy-to-light transitions, and light-to-heavy transitions that caused elements from a source block $X$ to be moved to a sink block $X^{\prime} \neq X$ (the latter have now been replaced with a modified split operation). These actions required $O(B) \mathrm{I} / \mathrm{O}$ operations to immediately update all affected pointers in $\mathcal{T}$ and $\mathcal{D}$. To deamortize these operations, we immediately move the elements from $X$ to $X^{\prime}$, but do not immediately update any pointers in $\mathcal{T}$ and $\mathcal{D}$. Instead, we create a pointer $p(X)$ from $X$ to $X^{\prime}$, allowing us to spread out the updates to $\mathcal{D}$ and $\mathcal{T}$ over many operations as follows.

We will ensure that any block $X$ need point to at most one block $X^{\prime}$ at any time; specifically, any time a split action or merge action causes items to move from block $X$ to block $X^{\prime}$, we will overwrite the old value of $p(X)$ with the new value. To clarify, when a block $X$ is sent to the free list as a result of a merge operation, it must maintain its pointer $p(X)$ throughout its time on the free list; it is only safe to overwrite $p(X)$ when items are once again moved from $X$ to another block $X^{\prime}$.

We will also ensure that no queue is ever moved more than once before its header in $\mathcal{T}$ and the records 
for all of its key-value pairs in $\mathcal{D}$ are brought up-to-date. Given this fact, if we ever follow a pointer from $\mathcal{T}$ or $\mathcal{D}$ to a block $X$, and the corresponding item is not in $X$, we need only look in $p(X)$ for the item as well.

To this end, we associate with each block $X^{\prime}$ in $\mathcal{S}$ a bit-array of length $O(B)$ indicating which items in $X^{\prime}$ have up-to-date pointers in $\mathcal{T}$ and $\mathcal{D}$. Any time items are moved into $X^{\prime}$ as a result of a split or merge action, we set the corresponding bits in the bit-array of $X^{\prime}$ to 0 , indicating these items are not up-to-date. Further, we modify the enqueue $(k, v)$ and remove $(k, v)$ operations such that if $(k, v)$ is stored in block $X$, then we update the pointers in $\mathcal{T}$ and $\mathcal{D}$ of up to 12 items in $X$ and 12 items from from $p(X)$ that are not up-to-date. We then mark these items as up-to-date. This requires only $O(1) \mathrm{I} / \mathrm{O}$ operations for each enqueue $(k, v)$ or remove $(k, v)$ function call.

We finally argue that each time items from a block $X$ to be moved to a block $X^{\prime}$, it is safe to overwrite $p(X)$ with a pointer to $X^{\prime}$. Indeed, we carefully argued above that all actions resulting in a movement of items from source block $X$ to sink block $X^{\prime}$ satisfy our required property. It is easy to see that this implies neither $X$ nor $X^{\prime}$ will be the source of another sink or merge until it is the target of at least $B / 12$ enqueue or remove operations. By that point, all items in $X$ (or $X^{\prime}$ ) and $p(X)$ (or $p\left(X^{\prime}\right)$ ) will be up-to-date, so it safe to overwrite $p(X)$ (or $p\left(X^{\prime}\right)$ ).

We obtain the following theorem.

Theorem 2. We can implement a location-aware multiqueue so that the remove $(x)$ and isMember $(x)$ operations each use $O(1) I / O s$, and the enqueue $(x, H)$ operation uses $O(1+t(N))$ expected I/Os, where $t(N)$ is the expected number of I/Os needed to perform an insertion in an external-memory cuckoo table of size $N$.

It should be clear from our description that, except for trivial cases (such as having only a constant number of elements), the space requirements of our multiqueue implementation is within a constant factor of the optimal. We have not attempted to optimize this factor, though there is some flexibility in the multiqueue operations (such as when to do a split) that would allow some optimization. We study these tradeoffs in Section 5 ,

\section{Combining Cuckoo Hashing and Location-Aware Multiqueues}

In this section, we describe how to construct an efficient external-memory multimap implementation by combining the data structures described above. The result is a cuckoo hash table in external memory so as to support constant expected-I/O insertions and optimal findAll and removeAll operations.

We store an external-memory cuckoo hash table, as described above, as our primary structure, $\mathcal{T}$, with each record pointing to a block in a multiqueue, $\mathcal{S}$, having an auxiliary dictionary, $D$, implemented as yet another external-memory cuckoo hash table. We then perform each of the operations of the multimap ADT as follows.

- $\operatorname{insert}(k, v)$ : To insert the key-value pair, $(k, v)$, we first perform a look up for $k$ in $\mathcal{T}$. If there is already a record for $k$ in $\mathcal{T}$, we increment its count. We then follow its pointer to the appropriate block $X$ in $\mathcal{S}$, (in the deamortized implementation, the queue for $k$ may reside in $p(X)$ rather than $X$ ), and add the pair $(k, v)$ to $\mathcal{S}$, as in the enqueue multiqueue method. Otherwise we insert $k$ into $\mathcal{T}$ with a null header record and count 1 and then add the pair $(k, v)$ to $\mathcal{S}$ as in the enqueue multiqueue method.

- isMember $(k, v)$ : This is identical to the isMember $(k, v)$ multiqueue operation.

- remove $(k, v)$ : To remove the key-value pair, $(k, v)$, from $C$, we perform a look up for $(k, v)$ in $\mathcal{D}$. If there is no record for $(k, v)$ in $\mathcal{D}$, we return an error condition. Otherwise, we follow this pointer to 


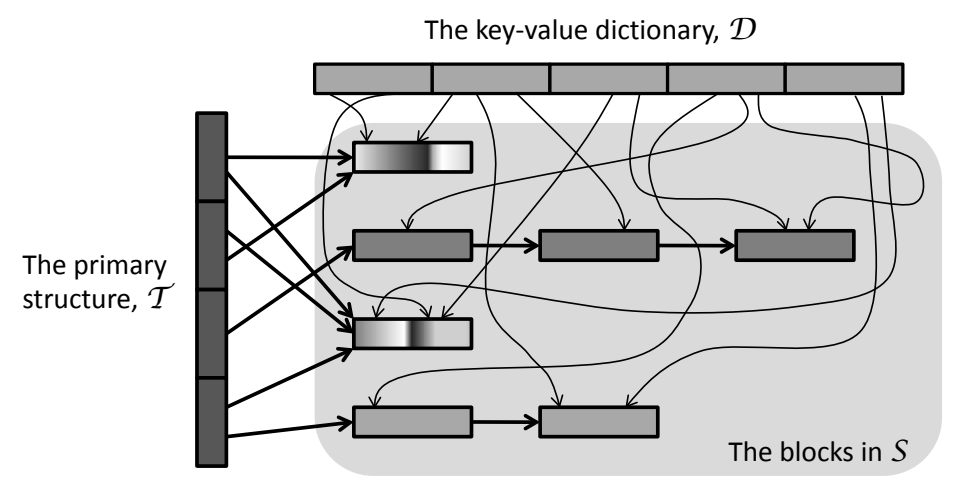

Figure 2: The external-memory multimap, online version.

the appropriate block $X$ of $\mathcal{S}$ holding the pair $(k, v)$ (in the deamortized implementation, if $(k, v)$ is not in $X$, we may have to look in $p(X)$ as well). We remove the pair $(k, v)$ from $\mathcal{S}$ and $\mathcal{D}$ as in the remove multiqueue method, and decrement its count.

- findAll $(k)$ : To return the set of all key-value pairs in $C$ having key equal to $k$, we perform a look up for $k$ in $\mathcal{T}$, and follow its pointer to the appropriate block of $\mathcal{S}$ (in the deamortized implementation, the queue for $k$ may reside in $p(X)$ rather than $X$ ). If this is a light queue, then we just return the items with key equal to $k$. Otherwise, we return the entire block and all the other blocks of this queue as well.

- removeAll $(k)$ : We give here a constant amortized time implementation, and explain in Section 4.1 how to deamortize this operation. To remove from $C$ all key-value pairs having key equal to $k$, we perform a look up for $k$ in $\mathcal{T}$, and follow its pointer to the appropriate block $X$ of $\mathcal{S}$ (in the deamortized implementation, the queue for $k$ may reside in $p(X)$ rather than $X$ ). If this is a light queue, then we remove from $X$ all items with key equal to $k$ and remove all affected pointers from $\mathcal{D}$; if this causes $X$ to become deficient, we perform a merge action or $d$-alteration action as in the remove multiqueue method. If this is a heavy queue, we walk through all blocks of this queue and remove all items from these blocks and return each block to the free list. We also remove all affected pointers from $\mathcal{D}$. Finally, we remove the header record for $k$ from $\mathcal{T}$, which implicitly sets the count of $k$ to zero as well. We charge, in an amortized sense, the work for all the I/Os to the insertions that added these key-value pairs to $C$ in the first place.

- $\operatorname{count}(k)$ : Return $n_{k}$, which we track explicitly for all keys $k$ in $\mathcal{T}$.

\subsection{Deamortizing removeAll $(k)$}

The removeAll $(k)$ operation of Section 4 required $O(1)$ amortized I/O operations in the worst case without altering the capacity of our structure. We now describe a deamortized implementation that also requires $O(1) \mathrm{I} / \mathrm{O}$ operations and does not alter the capacity. We perform a look up for $k$ in $\mathcal{T}$. If no record is found, we are done. Otherwise we follow its pointer to the header of its queue $Q$. We remove all items in $Q$ from $\mathcal{S}$, and set $k$ 's pointer in $\mathcal{T}$ to null. This completes the operation; notice we do not update any records in $\mathcal{D}$ 
at this time. Instead, we explain the modifications necessary to handle the existence of "spurious" pointers in $\mathcal{D}$ (i.e. pointers for $(k, v)$ pairs which were deleted in a removeAll operation) with an $O(1)$ increase in the $\mathrm{I} / \mathrm{O}$ cost of the $\operatorname{insert}(k, v)$, remove $(k, v)$, isMember $(k, v)$, and findAll $(k)$ operations.

First, we describe a function isSpurious $(k, v)$ that requires $O(1) \mathrm{I} / \mathrm{O}$ operations and determines whether an entry $(k, v)$ in $\mathcal{D}$ is spurious. isSpurious $(k, v)$ first peforms a look up in $\mathcal{D}$ for $(k, v)$. If no record for $(k, v)$ exists, we return false. Otherwise, we follow the pointer for $(k, v)$ to a block $X$ in $\mathcal{S}$ and search $X$ for $(k, v)$. If a record is found we return false. Otherwise, we follow the pointer $p(X)$ (described in Section 3.3) to a block $X^{\prime}$ and search for $(k, v)$ in $X^{\prime}$. If it is found, we return false, otherwise we return true.

We now describe how to modify the insertion method of our external-memory cuckoo hash table $\mathcal{D}$ so that the presence of spurious pointers does not decrease the table's capacity. First, when inserting a keyvalue pair $(k, v)$ into $D$, we begin by doing a look up in $\mathcal{D}$ for $(k, v)$. If a record for $(k, v)$ exists, we call isSpurious $(k, v)$. If this function returns false, we return an error condition. Otherwise, we remove the record for $(k, v)$ from $\mathcal{D}$ before proceeding. This ensures that at all times there is only one entry for each pair $(k, v)$ in $\mathcal{D}$.

Second, we modify the BFS-based insertion procedure of Theorem 1 as follows. For each bucket visited by the BFS, we call isSpurious $(k, v)$ for all pairs $(k, v)$ residing in the bucket. If this function returns true for any pair $(k, v)$, we delete $(k, v)$ from $\mathcal{D}$ and insert the new pair in its place. This ensures that no spurious entry in $\mathcal{D}$ ever prevents another entry from being inserted, i.e., the spurious entries will have no effect on the capacity of the table. Since the buckets in the cuckoo hashing algorithm of Theorem 1 have constant size, calling isSpurious $(k, v)$ on a bucket requires just $O(1) \mathrm{I} / \mathrm{O}$ operations.

With this in hand, we finally describe how to modify the insert $(k, v)$, remove $(k, v)$, isMember $(k, v)$, and findAll $(k)$ operations to handle the presence of spurious entries in $\mathcal{D}$ with only an $O(1)$ increase in the I/O complexity of each operation.

1. $\operatorname{insert}(k, v)$ : Works unmodified.

2. isMember $(k, v)$ : We call the previous implementation of isMember $(k, v)$ as well as the function checkSpurious $(k, v)$. We return true if and only if the former returns true and the latter returns false.

3. remove $(k, v)$ : We perform a look up for $(k, v)$ in $\mathcal{D}$. If none is found, we return an error condition. Otherwise, we call the function isSpurious $(k, v)$. If this returns true, we return an error condition. Otherwise, we call the old implementation of remove $(k, v)$.

4. findAll $(k)$ : Works unmodified.

We finally obtain the following theorem.

Theorem 3. One can implement the multimap ADT in external memory using $O(N / B)$ blocks of memory with I/O performance as shown in Table 1

\section{Experimental Results}

We performed extensive simulations of our algorithm in order to explore how various settings of the design parameters affect I/O complexity and space usage, for both our basic algorithm (Section 3.2) and our deamortized algorithm (Section 3.3).

We simulated a cache of size $M=512 \mathrm{~KB}$ with blocks of size $4 \mathrm{~KB}$. Our simulated cache used the least-recently used page replacement rule. When reporting the number of I/Os, we count only transfers from 
disk to cache; each such transfer is preceded by a transfer from cache to disk of the least recently used cache page, and we do not count this transfer in our reported values. We drew keys from a universe of size $2^{20} \approx 1$ million, using 4 bytes to store each key, and 8 bytes to store each value. We did not explicitly store queues as doubly-linked lists, but instead laid them out as arrays within their blocks, with a marker representing the end of one queue and the beginning of another; this allowed us to avoid storing expensive pointers for these lists. We used 4 bytes to represent all pointer values in $\mathcal{D}$ and $\mathcal{T}$. We did not charge for storing the counts associated with each key because we do not need to store these counts explicitly except to achieve $O(1) \mathrm{I} / \mathrm{O}$ operations for the count $(k)$ operation (and moreover we can achieve this by only storing explicit counts for heavy queues, as the count of a light queue $Q$ can be obtained in $\mathrm{O}(1)$ I/Os by finding $Q$ 's unique block in $\mathcal{S}$ via a lookup in $\mathcal{T}$ and then counting how many items $Q$ contains).

All results presented use random-walk cuckoo hashing with two hash functions and buckets capable of storing $4 \mathrm{KBs}$ of data; we found that using the partitioning technique of Theorem 1 to implement cuckoo hashing required slightly more space (and I/O complexity was comparable) because the hash tables had slightly smaller capacity. For our hash tables $\mathcal{D}$ and $\mathcal{T}$, we allotted a space overhead of $\varepsilon=0.07$; we found this was even more overhead than strictly necessary. We also ran a full set of experiments using three hash functions to implement cuckoo hashing, but found that two hash functions was sufficient due to the large bucket size; we found using two hash functions instead of three saved about $1 \mathrm{I} / \mathrm{O}$ per insert and remove operation. To capture realistic frequency distributions, which are often skewed, we generated all keys for insertions from a Zipfian distribution; in a Zipfian distribution with parameter $\alpha$, the frequency of the $k$ 'th most frequent item is proportional to $k^{-\alpha}$. The larger $\alpha$, the more skewed the frequency distribution.

Our goal was to identify the steady-state behavior of our data structure. In all experiments, we performed a sequence of 1 million insertions, followed by a sequence of 8 million alternating insert $(k, v)$ and remove $(k, v)$ operations. For each remove operation, the pair for removal was selected uniformly at random from the table.

\subsection{Basic Implementation}

Space usage results from the basic algorithm are shown in Figures 3(a) and 3(b), The vertical line represents the point at which we completed $2^{20} \approx 1$ million insertions and began alternating insertions and deletions. $\alpha$ denotes the Zipfian parameter, $B / \beta$ denotes the light-to-heavy queue transition threshold, and $B / \gamma$ is the deficiency parameter (i.e. blocks of size less than $B / \gamma$ are declared deficient). Notice we experiment with more aggressive settings of $\beta$ and $\gamma$ for $\alpha=1.1$.

Across all parameter settings, we achieved steady-state loads of between .33 and .39 , where we defined the load to be $S /\left(12 \times 2^{20}\right)$, where $S$ is the number of bytes used by pages not on the free list in our algorithm, and $12 \times 2^{20}$ is the minimum number of bytes required to explicitly store all $2^{20}$ key-value pairs in the structure. Notice with $4 \mathrm{~KB}$ blocks, $12 \times 2^{20}$ bytes corresponds to just over 3,000 blocks of memory.

$\mathrm{I} / \mathrm{O}$ statistics from representative settings of parameters are in Table 2 A smaller $\gamma$ results in improved space usage as a smaller $\gamma$ implies that we perform merge actions more aggressively. Similarly, a smaller $\beta$ implies we are more reluctant to tie up entire blocks devoted to a single heavy queue, and thus yields improved space usage.

In the basic algorithm, the average cost over all insert and remove operations is extremely low: about 3.5 I/Os per operation. However, as depicted in Table 2 the cost distribution is bimodal - the vast majority (over $99.9 \%$, except for $\gamma$ very close to 2) of operations require about $4 \mathrm{I} / \mathrm{Os}$, but a small fraction of operations require several hundred. The maximum number of I/Os ranges between 400 and 650 .

These high-cost operations are due to split and merge actions. The deamortized implementation displays substantially different behavior, with no operation requiring more than a few dozen I/Os (see Section 5.2). 


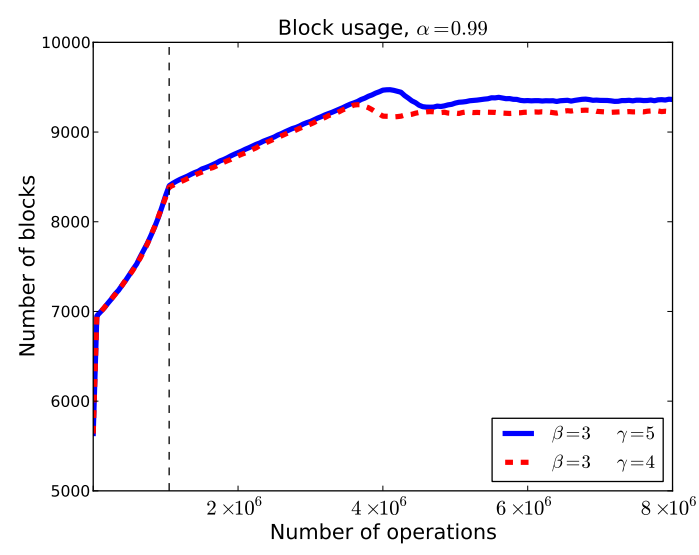

(a) Space usage for $\alpha=.99$.

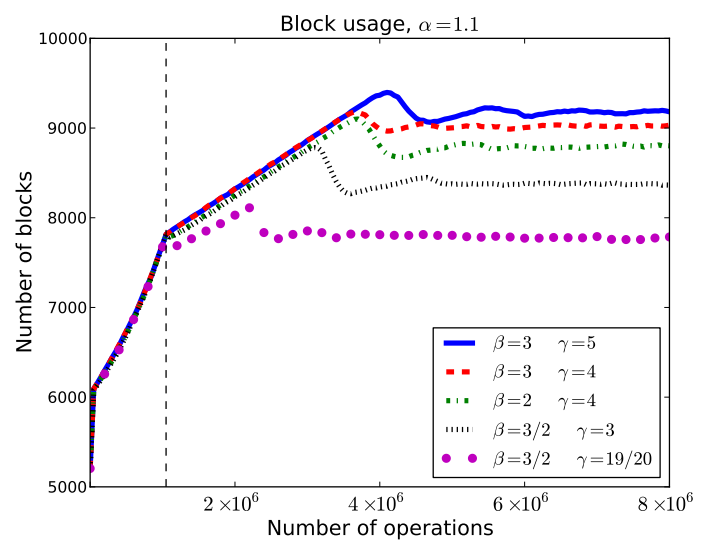

(b) Space usage for $\alpha=1.1$.

Figure 3: Results from simulations of an implementation of our basic (amortized) multimap algorithms.

Notice we tested parameter values for which the theoretical bounds on I/O complexity do not hold; for example, with $\gamma=19 / 10$, a merge may immediately follow a split.

(a) Overall

\begin{tabular}{|c|c|c|c|c|c|}
\hline$\alpha$ & $\beta$ & $\gamma$ & $\begin{array}{c}\text { Mean } \\
\text { I/Os }\end{array}$ & $\begin{array}{c}\text { Std Dev } \\
\text { I/Os }\end{array}$ & $\begin{array}{c}\text { Max } \\
\text { I/Os }\end{array}$ \\
\hline 0.99 & 3 & 5 & 3.53 & 4.24 & 639 \\
0.99 & 3 & 4 & 3.52 & 4.59 & 625 \\
\hline 1.10 & 3 & 5 & 3.17 & 4.29 & 398 \\
1.10 & 3 & 4 & 3.23 & 4.90 & 401 \\
1.10 & 2 & 4 & 3.20 & 5.27 & 403 \\
1.10 & $3 / 2$ & 3 & 3.25 & 6.73 & 534 \\
1.10 & $3 / 2$ & $19 / 10$ & 3.68 & 14.81 & 536 \\
\hline
\end{tabular}

(b) $\leq 15 \mathrm{I} / \mathrm{Os}$

\begin{tabular}{|c|c|c|c|c|c|}
\hline$\alpha$ & $\beta$ & $\gamma$ & $\begin{array}{c}\% \text { of } \\
\text { Ops }\end{array}$ & $\begin{array}{c}\text { Mean } \\
\text { I/Os }\end{array}$ & $\begin{array}{c}\text { Std Dev } \\
\text { I/Os }\end{array}$ \\
\hline 0.99 & 3 & 5 & 99.96 & 3.46 & 0.97 \\
0.99 & 3 & 4 & 99.96 & 3.44 & 0.96 \\
\hline 1.10 & 3 & 5 & 99.95 & 3.08 & 1.13 \\
1.10 & 3 & 4 & 99.94 & 3.12 & 1.14 \\
1.10 & 2 & 4 & 99.95 & 3.09 & 1.14 \\
1.10 & $3 / 2$ & 3 & 99.95 & 3.10 & 1.14 \\
1.10 & $3 / 2$ & $19 / 10$ & 99.83 & 3.09 & 1.13 \\
\hline
\end{tabular}

Table 2: I/O statistics for our basic (amortized) implementation (a) overall, (b) for operations requiring up to $15 \mathrm{I} / \mathrm{Os}$, and (c) for operations requiring more than $15 \mathrm{I} / \mathrm{Os}$. 


\subsection{Deamortized Implementation}

Figures 4(a) and 4(b) presents space usage results for the deamortized implementation, following the same protocol as the amortized experiments (Section 5.1). We achieved loads of about .33 to .35 for basic parameter values $(\gamma=4$ and $\gamma=5$ ). We also experimented with very high settings of $\gamma$, where we trade-off increased space usage for improved I/O complexity.

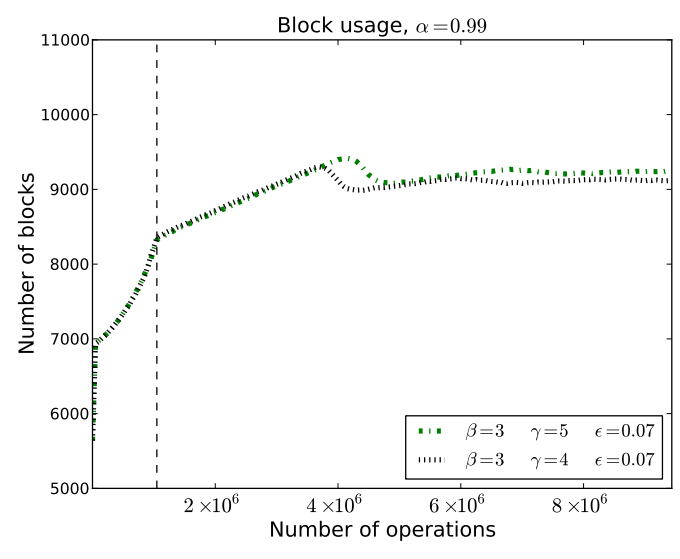

(a) Space usage for $\alpha=.99$.

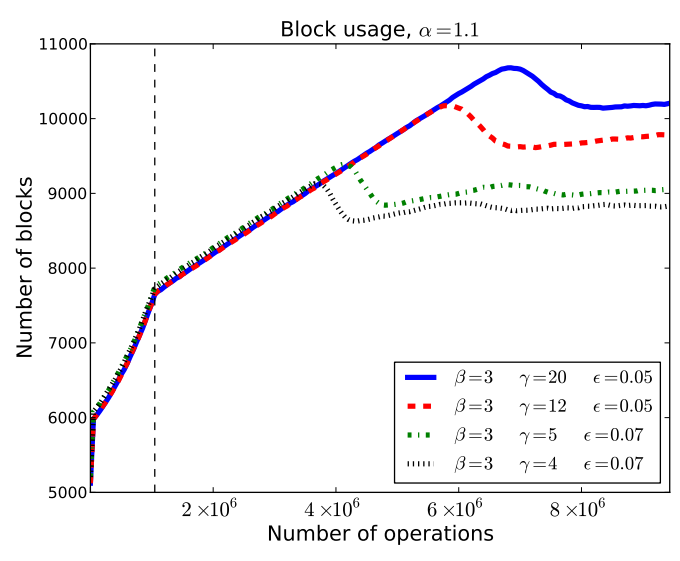

(b) Space usage for $\alpha=1.1$.

Figure 4: Results from simulations of an implementation of our deamortized multimap algorithms.

More interesting is the $\mathrm{I} / \mathrm{O}$ complexity of the deamortized implementation, shown in Table 3 . We see that in stark contrast to the bimodal cost distribution of the basic implementation, the deamortized implementation never requires more than a few dozen I/Os for any given operation. Moreover, even the average I/O complexity of the deamortized implementation is significantly better than that of the basic implementation, with an improvement of at least $0.5 \mathrm{I} / \mathrm{Os}$ per operation, for parameters where we have a direct comparison. We attribute much of this improvement to the modified split rule, which makes light-to-heavy queue transitions significantly less expensive. Note that the maximum number of I/Os for any operation in our deamortized experiments across all parameters, is at most 43 - an order of magnitude below the maximum for our basic algorithm.

In Table 3, we also display the breakdown in I/O complexity between inserts and remove operations. We see that removes are about twice as expensive as inserts; this is not unexpected. An insert requires a look up in $\mathcal{T}$, followed by loading the header page for the queue $Q$, an insert into $\mathcal{D}$, and then possibly a split. Due to the skewness of our input data, these first two steps can be free, as these pages are often already in the cache. A remove requires a look up in $\mathcal{D}$, followed by loading the appropriate page in $\mathcal{S}$, and then possibly a merge. In contrast to inserts, the first two steps are rarely free.

We note that we have tested our performance against a working commerical database product, which places key-value pairs in a hash table, but moves key-value pairs associated with a key to a B-tree when the number of values associated with a key becomes large. Preliminary tests suggest this approach yields a slightly smaller average number of memory accesses per operation, but in turn runs with significantly more memory. We expect that both implementations could be optimized significantly, and each approach might be preferable in different circumstances. 
(a) Overall

\begin{tabular}{|c|c|c|c|c|c|}
\hline$\alpha$ & $\beta$ & $\gamma$ & $\begin{array}{c}\text { Mean } \\
\text { I/Os }\end{array}$ & $\begin{array}{c}\text { Std Dev } \\
\text { I/Os }\end{array}$ & $\begin{array}{c}\text { Max } \\
\text { I/Os }\end{array}$ \\
\hline 0.99 & 3 & 5 & 2.96 & 1.75 & 42 \\
0.99 & 3 & 4 & 2.99 & 1.83 & 43 \\
\hline 1.10 & 3 & 20 & 2.60 & 1.66 & 41 \\
1.10 & 3 & 12 & 2.59 & 1.71 & 42 \\
1.10 & 3 & 5 & 2.66 & 1.96 & 42 \\
1.10 & 3 & 4 & 2.66 & 2.06 & 43 \\
\hline
\end{tabular}

(b) $\leq 15 \mathrm{I} / \mathrm{Os}$

\begin{tabular}{|c|c|c|c|c|c|}
\hline$\alpha$ & $\beta$ & $\gamma$ & $\begin{array}{c}\% \text { of } \\
\text { Ops }\end{array}$ & $\begin{array}{c}\text { Mean } \\
\text { I/Os }\end{array}$ & $\begin{array}{c}\text { Std Dev } \\
\text { I/Os }\end{array}$ \\
\hline 0.99 & 3 & 5 & 99.81 & 2.90 & 1.23 \\
0.99 & 3 & 4 & 99.78 & 2.92 & 1.24 \\
\hline 1.10 & 3 & 20 & 99.90 & 2.58 & 1.40 \\
1.10 & 3 & 12 & 99.88 & 2.56 & 1.39 \\
1.10 & 3 & 5 & 99.78 & 2.60 & 1.41 \\
1.10 & 3 & 4 & 99.73 & 2.58 & 1.41 \\
\hline
\end{tabular}

(d) Insert operations

\begin{tabular}{|c|c|c|c|c|c|}
\hline$\alpha$ & $\beta$ & $\gamma$ & $\begin{array}{c}\text { Mean } \\
\text { I/Os }\end{array}$ & $\begin{array}{c}\text { Std Dev } \\
\text { I/Os }\end{array}$ & Max \\
\hline 0.99 & 3 & 5 & 2.28 & 1.88 & 40 \\
0.99 & 3 & 4 & 2.32 & 2.00 & 42 \\
\hline 1.10 & 3 & 20 & 1.86 & 1.68 & 41 \\
1.10 & 3 & 12 & 1.85 & 1.76 & 42 \\
1.10 & 3 & 5 & 1.94 & 2.16 & 42 \\
1.10 & 3 & 4 & 1.94 & 2.32 & 41 \\
\hline
\end{tabular}

(c) $>15 \mathrm{I} / \mathrm{Os}$

\begin{tabular}{|c|c|c|c|c|c|}
\hline$\alpha$ & $\beta$ & $\gamma$ & $\begin{array}{c}\% \text { of } \\
\text { Ops }\end{array}$ & $\begin{array}{c}\text { Mean } \\
\text { I/Os }\end{array}$ & $\begin{array}{c}\text { Std Dev } \\
\text { I/Os }\end{array}$ \\
\hline 0.99 & 3 & 5 & 0.19 & 31.53 & 3.16 \\
0.99 & 3 & 4 & 0.22 & 31.44 & 3.23 \\
\hline 1.10 & 3 & 20 & 0.10 & 31.53 & 3.43 \\
1.10 & 3 & 12 & 0.12 & 31.38 & 3.26 \\
1.10 & 3 & 5 & 0.22 & 31.23 & 3.02 \\
1.10 & 3 & 4 & 0.27 & 31.12 & 3.05 \\
\hline
\end{tabular}

(e) Remove operations

\begin{tabular}{|c|c|c|c|c|c|}
\hline$\alpha$ & $\beta$ & $\gamma$ & $\begin{array}{c}\text { Mean } \\
\text { I/Os }\end{array}$ & $\begin{array}{c}\text { Std Dev } \\
\text { I/Os }\end{array}$ & Max \\
\hline 0.99 & 3 & 5 & 3.80 & 1.09 & 42 \\
0.99 & 3 & 4 & 3.82 & 1.12 & 43 \\
\hline 1.10 & 3 & 20 & 3.53 & 1.08 & 40 \\
1.10 & 3 & 12 & 3.52 & 1.09 & 42 \\
1.10 & 3 & 5 & 3.57 & 1.15 & 42 \\
1.10 & 3 & 4 & 3.55 & 1.18 & 43 \\
\hline
\end{tabular}

Table 3: I/O statistics for the deamortized implementation, for (a) overall, (b) for operations requiring up to $15 \mathrm{I} / \mathrm{Os}$, (c) for operations requiring more than $15 \mathrm{I} / \mathrm{Os,}$ (d) for insert operations, and (e) for remove operations.

\section{Conclusion}

We have described an efficient external-memory implementation of the multimap ADT, which generalizes the inverted file data structure that is useful for supporting search engines. Our methods are based on new expected-time bounds for performing updates in block-based cuckoo hash tables as well as an externalmemory multiqueue data structure. In addition to proving theoretical bounds on the I/O complexity of our implementation, we demonstrated experimentally that our data structure is able to trade off constant factors in space against the time to perform operations in well-understood ways.

One direction for future work is to consider efficient in-memory algorithms for multimaps, an area that seems to not have been given significant attention. Another natural direction would be to derive improved high-probability bounds for block-based cuckoo hash tables. In particular, improved analysis of random walk cuckoo hashing in this setting is worthwhile. These are natural extensions of open problems in the theory of cuckoo hashing. 


\section{Acknowledgments}

We thank Margo Seltzer for several helpful discussions.

\section{References}

[1] A. Aggarwal and J. S. Vitter. The input/output complexity of sorting and related problems. Commun. ACM, 31:1116-1127, 1988.

[2] D. A. Alcantara, A. Sharf, F. Abbasinejad, S. Sengupta, M. Mitzenmacher, J. D. Owens, and N. Amenta. Real-time parallel hashing on the GPU. ACM Trans. Graph., 28:154:1-154:9, 2009.

[3] D. K. Blandford and G. E. Blelloch. Compact dictionaries for variable-length keys and data with applications. ACM Transactions on Algorithms, 4(2), 2008.

[4] S. Büttcher and C. L. A. Clarke. Indexing time vs. query time: trade-offs in dynamic information retrieval systems. In Proc. of 14th ACM Conf. on Information and Knowledge Management (CIKM), pages 317-318. ACM, 2005.

[5] S. Büttcher, C. L. A. Clarke, and B. Lushman. Hybrid index maintenance for growing text collections. In Proc. of 29th ACM SIGIR Conf. on Research and Development in Information Retrieval (SIGIR), pages 356-363. ACM, 2006.

[6] D. Cutting and J. Pedersen. Optimization for dynamic inverted index maintenance. In 13th ACM SIGIR Conf. on Research and Development in Information Retrieval, SIGIR '90, pages 405-411. ACM, 1990.

[7] S. Dehaene. The neural basis of the Weber-Fechner law: a logarithmic mental number line. Trends in Cognitive Sciences, 7(4):145-147, 2003.

[8] M. Dietzfelbinger and C. Weidling. Balanced allocation and dictionaries with tightly packed constant size bins. Theoretical Computer Science, 380:47-68, 2007.

[9] R. Guo, X. Cheng, H. Xu, and B. Wang. Efficient on-line index maintenance for dynamic text collections by using dynamic balancing tree. In Proc. of 16th ACM Conf. on Information and Knowledge Management (CIKM), CIKM '07, pages 751-760. ACM, 2007.

[10] S. Hecht. The visual discrimination of intensity and the Weber-Fechner law. Journal of General Physiology, 7(2):235-267, 1924.

[11] D. E. Knuth. Sorting and Searching, volume 3 of The Art of Computer Programming. Addison-Wesley, Reading, MA, 1973.

[12] N. Lester, A. Moffat, and J. Zobel. Efficient online index construction for text databases. ACM Trans. Database Syst., 33:19:1-19:33, September 2008.

[13] N. Lester, J. Zobel, and H. Williams. Efficient online index maintenance for contiguous inverted lists. Inf. Processing \& Management, 42(4):916-933, 2006.

[14] R. W. Luk and W. Lam. Efficient in-memory extensible inverted file. Information Systems, 32(5):733-754, 2007. 
[15] M. Mitzenmacher and E. Upfal. Probability and Computing: Randomized Algorithms and Probabilistic Analysis. Cambridge University Press, New York, NY, USA, 2005.

[16] M. Mitzenmacher and S. Vadhan. Why simple hash functions work: exploiting the entropy in a data stream. In Proc. of the 19th Annual ACM-SIAM Symp. on Discrete Algorithms, pages 746-755, 2008.

[17] D. R. Musser and A. Saini. The STL Tutorial and Reference Guide: C++ Programming with the Standard Template Library. Addison Wesley Longman Publishing Co., Inc., Redwood City, CA, USA, 1995.

[18] R. Pagh and F. Rodler. Cuckoo hashing. Journal of Algorithms, 52:122-144, 2004.

[19] R. Panigrahy. Efficient hashing with lookups in two memory accesses. In Proc. of the 16th Annual ACM-SIAM Symp. on Discrete Algorithms, pages 830-839, 2005.

[20] R. Panigrahy. Hashing, Searching, Sketching. Ph.D. thesis, Dept. of Computer Science, Stanford University., 2006.

[21] E. Verbin and Q. Zhang. The limits of buffering: a tight lower bound for dynamic membership in the external memory model. In STOC, pages 447-456, 2010.

[22] J. S. Vitter. External sorting and permuting. In M.-Y. Kao, editor, Encyclopedia of Algorithms. Springer, 2008.

[23] J. Zobel and A. Moffat. Inverted files for text search engines. ACM Comput. Surv., 38, July 2006. 